Banken und Sparkassen am strategischen Scheideweg

\author{
DR. BENÖLKEN + PARTNER GmbH \\ Unternehmensberatung \\ Willi-Becker-Allee 11. 4000 Düsseldorf 1 \\ Telefon: (02 11) 772013 , Telefax: (02 11) 773622
}


CIP-Titelaufnahme der Deutschen Bibliothek

Banken und Sparkassen am strategischen Scheideweg : eine

Studie / von Dr. Benölken + Partner GmbH. - Wiesbaden :

Gabler, 1991

NE: Dr. Benölken Partner < Düsseldorf >

Der Gabler Verlag ist ein Unternehmen der Verlagsgruppe Bertelsmann International.

(C) Springer Fachmedien Wiesbaden 1991

Ursprünglich erschienen bei Betriebswirtschaftlicher Verlag Dr. Th. Gabler GmbH, Wiesbaden 1991.

Lektorat: Karlheinz Müssig

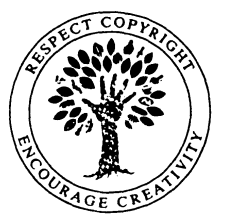

Das Werk einschließlich aller seiner Teile ist urheberrechtlich geschützt. Jede Verwertung außerhalb der engen Grenzen des Urheberrechtsgesetzes ist ohne Zustimmung des Verlags unzulässig und strafbar. Das gilt insbesondere für Vervielfältigungen, Übersetzungen, Mikroverfilmungen und die Einspeicherung und Verarbeitung in elektronischen Systemen. 
Eine Reihe von Finanzdienstleistungskunden, Sparkassen, Genossenschaftsbanken, Institute mit begrenzter regionaler Geschäftstätigkeit im privaten Bereich sowie auch anderen Finanzdienstleistungen sehen sich in ihren strategischen Überlegungen mit dem Phänomen "EG-Binnenmarkt 1992" konfrontiert:

- Können sie noch allein im Wettbewerb bestehen?

- Sollen sie sich zu größeren Einheiten zusammenschließen?

- Welche strategische Alternative stellt die zentrale Überlebensfrage dar?

In einer Reihe von Projekten hatten wir Gelegenheit, gerade auch im sog. "gehobenen Mittelstand" des Finanzdienstleistungsgewerbes zu solchen Fragestellungen zu argumentieren und mögliche strategische Erfolgsfaktoren gemeinsam mit dem Kunden zu erarbeiten. Die daraus gewachsenen Ergebnisse haben uns motiviert, dieses Thema grundsätzlich aufzuarbeiten. Dabei trug zu einem gerüttelten Maße die konkrete Projektarbeit zu einer empirischen Basis bei, ergänzt um eine empirische Umfrage bei "einschlägigen" regional tätigen Banken in unterschiedlichen Institutsgruppen. Über 90 Institute haben sich mit positiver Rückmeldung daran beteiligt, so daß Szenarien, die konkreten Projekterfahrungen sowie die Umfrageergebnisse eine hervorragende Basis für die Erarbeitung grundsätzlicher strategischer Ansatzpunkte und Maßnahmen bilden.

Diese Studie wird, so hoffen wir, auch Finanzdienstleistern helfen, die jeweils konkrete Strategie für das eigene Haus, die unverwechselbar ist, zu finden und somit auch unter EG-Binnenmarkt-Bedingungen in den 90er Jahren erfolgreich im jeweiligen Marktsegment zu agieren.

Sollte die Praxis in dieser Studie eine wesentliche Hilfe für die strategische Arbeit sein, so wäre das Ziel der Studie erfüllt und es wäre für die "Bearbeiter" der schönste Lohn.

Gern sind wir bereit, im Dialog mit dem einzelnen Kreditinstitut auch für institutsspezifische Szenarien und Workshops als Sparringspartner zur Verfügung zu stehen.

DR. BENÖLKEN + PARTN ER GmbH 


\section{Gliederung (1)}

A. Binnenmarktstrategie für mittlere Banken -

Seite

Mode oder strategische Notwendigkeit?

B. Die wichtigsten Entwicklungstrends im bankbetrieblichen Umfeld

1. Wesentliche Entwicklungstendenzen auf den Finanzdienstleistungsmärkten

1.1 Fortsetzung und Beschleunigung der Internationalisierungstendenzen

1.2 Auflösung traditioneller Angebotsstrukturen

1.2.1 Tendenz zum Universalbanksystem

1.2.2 Verbund, Konzern, Betriebsgröße

1.2.3 Zunehmende Angebotsvielfalt Allfinanzkonzepte

2. Veränderungen der Ausgangssituation durch Einführung des gemeinsamen Binnenmarktes

2.1 Der gemeinsame Markt: Entwicklungstendenzen und Erwartungen

2.2 Veränderungen in der rechtlichen Ausgangssituation

2.2.1 Die künftige Geld- und Währungspolitik

2.2.2 Die Harmonisierung der Steuersysteme

2.2.3 Die Vorhaben im Gesellschaftsrecht

2.3 Veränderungen in der wirtschaftlichen Ausgangssituation

2.3.1 Der EG-Binnenmarkt: Großes Volumen und inhomogene Strukturen

2.3.2 Nationale Verbraucher versus EuroKonsumenten

2.3.3 Europäisierung und regionale Strukturveränderungen im Binnenmarkt 
3. Veränderungen des bankbetrieblichen Handlungsrahmens im europäischen Binnenmarkt

Seite

3.1 Veränderungen der bankrechtlichen Rahmenbedingungen

3.1.1 Die Eigenmittelrichtlinie

3.1.2 Die Solvabilitätsrichtlinie

3.1.3 Die Investmentrichtlinie

42

3.1.4 Die Insiderrichtlinie

43

3.1.5 Sonstige bankrechtliche Harmonisierungsmaßnahmen

3.2 Strukturelle Veränderungen in ausgewählten Bankenmärkten in Europa

3.2.1 Entwicklungstendenzen im französischen Banksystem

3.2.2 Entwicklungstendenzen im spanischen Banksystem

3.2.3 Entwicklungstendenzen im italienischen Banksystem

3.2.4 Entwicklungstendenzen im britischen Banksystem

3.2.5 Entwicklungstendenzen im österreichischen Banksystem

4. Auswirkungen des Binnenmarktes auf die Nachfrage- und Wettbewerbsstrukturen am Bankenmarkt

4.1 Wesentliche Veränderungen im Privatkundenmarkt

4.2 Wesentliche Veränderungen im Firmenkundenmarkt

4.2.1 Die wichtigsten Konsequenzen der EGHarmonisierung für die Firmenkunden 


\section{Gliederung (3)}

Seite

4.2.2 Spezielle Auswirkungen der EG-Harmonisierung auf ausgewählte Branchen

- Die Agrarbranche

- Die Elektronikbranche

- Die Textilbranche

- Die Nahrungs- und Genu Bmittelbranche

- Die Automobilbranche

- Die Baubranche

- Die Maschinenbaubranche

- Die Straßengüterverkehrbranche 97

- Die Einzelhandelsbranche 99

- Sonstige Branchen

4.3 Auswirkungen des Binnenmarktes auf die geschäftspolitischen Handlungsparameter der Banken 106

C. Die Ergebnisse der empirischen Analysen

1. Gang der Untersuchung und empirische Basis

2. Das Informationsverhalten der Kreditinstitute

2.1 Die Bedeutung verschiedener Informationsquellen

2.2 Konzepte zur Informationsaufbereitung und Informationsweitergabe

3. Alternative Strategien zur zukünftigen Marktbearbeitung

3.1 Die generelle Einschätzung der künftigen Markt- und Wettbewerbssituation

3.2 Die Zielgruppenkonzepte

3.3 Die Vertriebskonzepte

3.4 Einschätzung der geschäftspolitischen Reaktionsmöglichkeiten 
4. Ausgewählte Fragestellungen kleinerer und mittlerer Banken im EG-Binnenmarkt

4.1 Beurteilung der optimalen Betriebsgröße bei Kreditinstituten

4.2 Beurteilung von Handlungsalternativen zur Lösung von Kosten- und Strukturproblemen

4.3 Beurteilung von Optionen für Auslandsaktivitäten

4.4 Die Bedeutung des Wettbewerbsfaktors Bankmitarbeiter

5. Resümée

D. Strategische Optionen im EG-Binnenmarkt für mittlere Banken

1. Zielgruppenstrategien

2. Produktstrategien

$\begin{array}{ll}\text { 3. Vertriebsstrategien } & 149\end{array}$

4. Personalstrategien 155

5. Betriebsbereichsstrategien 158

5.1 Outsourcing-Strategien für Kreditinstitute

5.2 Etablierung eines umfassenden RiskManagement

E. Weiterführende Literaturempfehlungen 